\title{
Stability Analysis for Network Coded Multicast Cell with Opportunistic Relay
}

\author{
Zhihui Shu*, Wen Chen*†, Xinbing Wang*, and Xiaoting Yang $\ddagger$ \\ *Department of Electronic Engineering,Shanghai Jiaotong University, China 200240; \\ $\dagger$ SKL for Mobile Communications, Southeast University, China 211189; \\ $\ddagger$ Department of Electrical and Computer Engineering, University of Denver, USA \\ Email: $\{$ jxby001;wenchen;xwang8\} @ sjtu.edu.cn
}

\begin{abstract}
In this paper, we propose an opportunistic relay with network coding for multcast cell. Specifically, we propose three strategies for the opportunistic relay. By analyzing the stability regions for the three proposed strategies, we find that the two strategies with network coding outperform that without network coding in terms of stability region. In addition, the strategy with opportunistic network coding outperforms that with relatively static network coding. Finally, simulation results validate our theoretical predictions.

Index Terms-Multicast network, opportunistic relay, network coding, stability region.
\end{abstract}

\section{INTRODUCTION}

Cognitive radio network has been a new technology in wireless communication that improves utilization of limited spectral resources as the demand for wireless spectrum increases rapidly in recent years [1]. The limited available spectrum and the inefficiency in the spectrum usage necessitate a new communication paradigm to exploit the existing wireless spectrum opportunistically [2], or in spectrum sharing [3]. A basic cognitive network with only one primary user and one secondary user has been investigated in [4], where the authors analyze the cognitive user's capacity without reducing the achievable rate of primary users in information-theoretic view. Joint physical and MAC layer analysis in cognitive radio network is considered in [5]. The authors demonstrate that the gains promised by cooperation at the physical layer can be leveraged to the MAC layer. The authors also address the stability region of the primary users in multiple access channel and delay analysis of the primary users.

Network coding is originally introduced to achieve the network multicast capacity in the wireline systems [6]. It has been extended to the wireless networks in [7] and [8]. In [9], the authors investigate the wireless multicast networks with regenerative network coding, and propose a power control scheme to achieve the full diversity. Most of these previous works on network coding assume that the source nodes always transmit packets. However, this is not always the case. For example, most of the sources in the wireless networks are bursty. Sometimes, they transmit data, while keep silent in some other times. Traffic dynamics at the primary user is considered in [10], where the activity of the primary user is modeled as Poisson process. The opportunistic spectrum access protocol is proposed in [11], where the secondary user can use the free time slots when the primary user isn't transmitting. In [12], the authors investigate the power allocation schemes in cognitive radio networks with network coding. However, the authors did not analyze how cognitive users cooperate with the primary users. The authors did not yet analyze the network coding strategy in MAC layer or the QoS problems either.

In this paper, we leverage network coding in network coded multicast networks for system throughput improvement. When the packets from the source nodes are transmitted unsuccessfully to destinations, but successfully to the relay, the packets can be stored in the relay. But these packets have different types. Some are transmitted unsuccessfully to both destinations, and some are transmitted unsuccessfully to only one destination. In order to perform network coding, we should carefully schedule these packets to decide which packets can be network coded and which packets should be transmitted firstly.

In this paper, we will focus on the regenerative network coding where mixed signals are decoded at opportunistic relay and retransmitted to the destinations. We propose a dynamic network coding scheme, and demonstrate that our strategy with network coding can significantly improve throughput region compared to the strategy without network coding in the opportunistic relay of a multicast cell. In addition, the performance will be different for different strategies applied on the relay. Our contribution in this paper is: i) focusing on a physical layer view of the outage probability and applying an opportunistic relay protocol with network coding to a multicast cell, ii) investigating the QoS improvement and throughput of the multicast cell.

\section{SYSTEM MODEL}

We consider a multicast cell consisting of two users and an opportunistic relay node (see Fig. 1). The two source nodes are denoted by $S_{1}$ and $S_{2}$, the two destination nodes are denoted by $D_{1}$ and $D_{2}$, and the relay node is denoted by $R$. In our model, we assume that the relay consists of three queues $R_{0}, R_{1}$ and $R_{2} . S_{1}$ and $S_{2}$ transmit according to the TDMA protocol. Both source nodes need to broadcast information to both destinations successfully.

Each source node transmits one packet during one time slot sequentially. When the queue is empty, the time slot is 


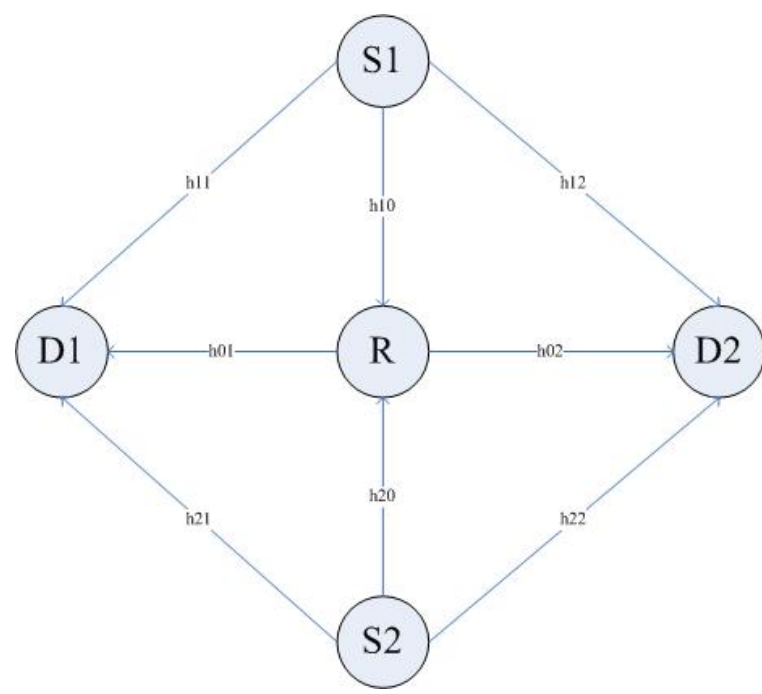

Fig. 1. The multicast cell with opportunistic network coded relay.

regarded to be free. When $S_{1}$ or $S_{2}$ transmits to $D_{1}, D_{2}$ and $R$ in one time slot, there are several transmission cases which are summarized in the following forms:

\begin{tabular}{|l|l|l|l|l|l|l|l|l|}
\hline & 1 & 2 & 3 & 4 & 5 & 6 & 7 & 8 \\
\hline$D_{1}$ & $Y$ & $Y$ & $N$ & $Y$ & $N$ & $N$ & $Y$ & $N$ \\
\hline$D_{2}$ & $Y$ & $Y$ & $N$ & $N$ & $Y$ & $N$ & $N$ & $Y$ \\
\hline$R$ & $Y$ & $N$ & $Y$ & $Y$ & $Y$ & $N$ & $N$ & $N$ \\
\hline Type & \multicolumn{2}{|l|}{ Success } & $F_{R_{0}}$ & $F_{R_{1}}$ & $F_{R_{2}}$ & $F_{0}$ & $F_{1}$ & $F_{2}$ \\
\hline
\end{tabular}

where "Y" means success and "N" means failure. The notations $F_{R_{0}}, F_{R_{1}}, F_{R_{2}}, F_{0}, F_{1}$ and $F_{2}$ are the transmission types to be defined.

Case 1 and case 2 are considered as successful transmissions since both $D_{1}$ and $D_{2}$ successfully received information from the source node. In case 3 , the transmitted packet is called $F_{R_{0}}$-packet and stored in the $R_{0}$. In case 4 , the transmitted packet is called $F_{R_{1}}$-packet and stored in $R_{1}$. In case 5 , the transmitted packet is called $F_{R_{2}}$-packet and stored in $R_{2}$. For the above cases, the source node doesn't need an additional transmission. However, case 6-8 are regarded as unsuccessful transmissions since at most one destination node received information from the source. In these cases, the source node needs another transmission for the transmitted packet. The transmitted packet in case 6-8 are called $F_{0^{-}}, F_{1^{-}}$and $F_{2^{-}}$ packet respectively. Besides, the initial packet to be transmitted is also called $F_{0}$-packet.

When the relay decodes the packet successfully (unsuccessfully), it will send an ACK (NACK) message to the source. So the source knows the success (failure) of the transmission to the relay. When the source node transmits a packet to the destination nodes, the destinations will broadcast ACK or NACK messages to both the source nodes and relay according to the decoding status. Therefore, the source nodes and the relay will know success (failure) of this transmission. Similarly, when the relay transmits packet to the destination nodes, the destination nodes will also send ACK or NACK messages to the relay. In the following, we introduce the dynamic network coding strategies based on the relay queue sizes, which allow different priorities (in terms of order of transmission) to the three kinds of packets $F_{R_{0}}, F_{R_{1}}, F_{R_{2}}$.

Strategy 1: When the slot of source node is sensed free, the relay can transmit the packet to the destination. The transmitting strategy in the relay is as follows: the relay node first transmits the $F_{R_{0}}$-packet if $R_{0}$ is not empty. Otherwise, Only if $R_{1}$ is empty, the relay transmits the $F_{R_{2}}$-packet to $D_{1}$; only if $R_{2}$ is empty, the relay transmits the $F_{R_{1}}$ packet to $D_{2}$; if none of $R_{1}$ and $R_{2}$ is empty, the relay performs network coding for the packets in $R_{1}$ and $R_{2}$ and transmits $F_{R_{1}} \oplus F_{R_{2}}$ to the destination; if $R_{1}$ and $R_{2}$ are both empty, the relay keeps idle.

Strategy 2: The transmitting strategy in the relay is as follows: the relay node first transmits $F_{R_{1}} \oplus F_{R_{2}}$, if none of $R_{1}$ and $R_{2}$ is empty; the relay transmits $F_{R_{1}}\left(F_{R_{2}}\right)$ to $D_{2}$ ( $\left.D_{1}\right)$ if only $R_{2}\left(R_{1}\right)$ is empty; the relay transmits $F_{R_{0}}$ to the destination only if both $R_{1}$ and $R_{2}$ are empty; the relay keeps idle if $R_{0}, R_{1}$ and $R_{2}$ are all empty.

Strategy 3: Combine the three queues into one queue, and all the three kind packets $F_{R_{0}}, F_{R_{1}}$ and $F_{R_{2}}$ are put into the queue sequentially. At each sensed empty time slot, the packets in the relay are transmitted according to the FIFO (First In First Out) rule.

In our queueing model, the time is slotted, and in each time slot there is no more than one packet to be transmitted (all the packets are assumed to have the same packet length). The packet arrival processes at $S_{1}$ and $S_{2}$ are both independent and stationary with rate $\lambda_{1}$ and $\lambda_{2}$ (packets per slot) respectively. Both source nodes access the channel according to the TDMA protocol. Let $\Omega=\left[\omega_{1}, \omega_{2}\right]$ denote a time-sharing vector, where $\omega_{i}$ denotes the probability that time slot is allocated to the source node $i$.

Stability is an important performance metric in the queueing analysis [15]. In this paper, the stability is defined as in [14], i.e.,

$$
\lim _{t \rightarrow \infty} P\left[Q_{i}(t)=0\right]>0,
$$

where $Q_{i}(t)$ means the length of the waiting packets in the $i$ th queue at time $t$. If the arrival and departure rates of a queueing system are stationary, then Loyne's theorem can be used to check the stability of the queue. The $i$ th queue of the source node is considered to be stable if the average arrival rate is less than the average service rate. If the average arrival rate is greater than the average service rate, the queue is unstable. We don't consider the case that the average arrival rate is equal to the average service rate as there is a possibility of subtle limiting behavior.

All wireless channels between any two nodes in the system are modeled as independent stationary Rayleigh flat-fading channels with additive Gaussian noise. The channel coefficients $h_{i j}^{t}$ is modeled as i.i.d zero mean, circularly symmetric complex Gaussian random process with unit variance. The node transmits with normalized power $P=1$. without loss of 
generality, the noise at all the receiver is modeled as i.i.d zero mean, circularly symmetric complex Gaussian random process with unit variance. We also assume that the channel fading coefficients are known only to the corresponding receivers, but not known to the transmitters.

The instant received SNR of a signal transmitted from node $i$ to node $j$ can be shown to be $\operatorname{SNR}_{i j}=\frac{\gamma_{i j}\left|h_{i j}\right|^{2} P_{i}}{N_{0}}$, where $\left|h_{i j}\right|^{2}$ denotes the magnitude channel gain square which has an exponential distribution with unit mean. $\gamma_{i j}$ denotes the average channel power gain due to the shadowing and path loss.

The transmission of a given packet is regarded to be successful if the received SNR is above a given threshold $\beta$, which can be chosen according to the QoS requirement. Therefore, the probability of outage of the transmission can be shown to be $P_{\text {out }, i j}=P\left[\operatorname{SNR}_{i j}<\beta\right]=1-\exp \left(-\frac{\beta}{\gamma_{i j}}\right)$, which follows from the exponential distribution of the received SNR. So the success probability of the transmission at SNR threshold $\beta$ is

$$
P_{i, j}=\exp \left(-\frac{\beta}{\gamma_{i j}}\right) .
$$

\section{Stability ANAlysis for Strategy 1}

As there are three kinds of packets in the sources $S_{1}$ and $S_{2}$, we should study the arrival and service rate of the three kind packets to analyze the stability of the sources' queue.

\section{A. Stability condition for $S_{1}$ and $S_{2}$}

Consider only the $F_{0}$ packet in $S_{1}$. Similar in [5] and [14], the queue size (in packets) of $S_{1}$ evolves as

$$
Q_{1}(t)=\left(Q_{1}(t-1)-Y_{1,0}(t)\right)^{+}+X_{1,0}(t),
$$

where $X_{1,0}(t)$ is the stationary process representing the number of arrivals of the $F_{0}$ packet, $Y_{1,0}(t)$ is the service process of $F_{0}$ packet, and $(x)^{+}=\max (x, 0)$. For simplicity, we will omit $t$ in the following analysis. We also assume that the queue size is measured at the beginning of the slot. Let $A_{1}$ be the time slot allocated to $S_{1}$. From the system model, we know that the probability of $\left\{A_{1}=1\right\}$ is $\omega_{1}$. Let $O_{S_{1} D_{1}}$ denote the event that $S_{1}$ transmits successfully to $D_{1}$, which probability is assumed to be $P_{S_{1} D_{1}}$ and can be determined by (2). Similarly, $O_{S_{1} D_{2}}$ and $O_{S_{1} R}$ denote the events that $S_{1}$ transmits successfully to $D_{2}$ and $R$ respectively, which probabilities are assumed to be $P_{S_{1} D_{2}}$ and $P_{S_{1} R}$ respectively, and determined by (2). From the system model, the service process of the $F_{0}$ packet in $S_{1}$ is $Y_{1,0}=\chi_{A_{1} \cap\left(O_{S_{1} D_{1}} \cup O_{S_{1} D_{2}} \cup O_{S_{1} R}\right)}$, where $\chi_{\{\cdot\}}$ is the indicator function. So the service rate of the $F_{0}$-packet in $S_{1}$ is

$$
\mu_{1,0}=\omega_{1}\left(1-P_{1,3}\right),
$$

where $P_{1,3}=\left(1-P_{S_{1} D_{1}}\right)\left(1-P_{S_{1} D_{2}}\right)\left(1-P_{S_{1} R}\right)$.

When $S_{1}$ transmits a packet only successfully to $D_{1}$, the packet in $S_{1}$ becomes the $F_{1}$-packet. The arrival process of the $F_{1}$-packet in $S_{1}$ is $X_{1,1}=$

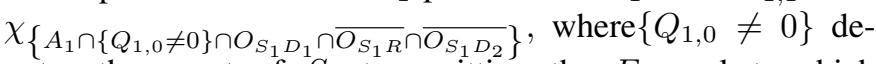
notes the event of $S_{1}$ transmitting the $F_{0}$ packet, which probability, by Little's theorem, is $\frac{\lambda_{1}}{\omega_{1}\left(1-P_{1,3}\right)}$. So the arrival rate of the $F_{1}$ packet in $S_{1}$ is

$$
\lambda_{1,1}=E\left[X_{1,1}\right]=\frac{\lambda_{1} P_{S_{1} D_{1}}}{1-P_{1,3}}\left(1-P_{S_{1} R}\right)\left(1-P_{S_{1} D_{2}}\right) .
$$

Next, we consider the service process of the $F_{1}$-packet in $S_{1}$. When the $F_{1}$-packet is transmitted successfully to $R$ or $D_{2}$, it can leave the source node. So the service process is $Y_{1,1}=A_{1} \cap\left(O_{S_{1} R} \cup O_{S_{1} D_{2}}\right)$. Then the service rate for $F_{1^{-}}$ packet in $S_{1}$ is

$$
\mu_{1,1}=\omega_{1}\left(P_{S_{1} R}+P_{S_{1} D_{2}}-P_{S_{1} R} P_{S_{1} D_{2}}\right) .
$$

Similarly, when $S_{1}$ transmits the packet only successfully to $D_{2}$, the packet becomes the $F_{2}$-packet in $S_{1}$. Then the arrival rate of the $F_{2}$ packet in $S_{1}$ is

$$
\lambda_{1,2}=\frac{\lambda P_{S_{1} D_{2}}}{1-P_{1,3}}\left(1-P_{S_{1} R}\right)\left(1-P_{S_{1} D_{1}}\right),
$$

and the service rate of the $F_{2}$-packet in $S_{1}$ is

$$
\mu_{1,2}=\omega_{1}\left(P_{S_{1} R}+P_{S_{1} D_{1}}-P_{S_{1} R} \cdot P_{S_{1} D_{1}}\right) .
$$

From the Loynes' theorem, the stability condition for $S_{1}$ is

$$
\frac{\lambda_{1}}{\mu_{1}}=\frac{\lambda_{1}}{\mu_{1,0}}+\frac{\lambda_{1,1}}{\mu_{1,1}}+\frac{\lambda_{1,2}}{\mu_{1,2}}<1,
$$

where $\mu_{1}$ denotes the average service rate of $S_{1}$. Similarly, the stability condition for $S_{2}$ is

$$
\frac{\lambda_{2}}{\mu_{2}}=\frac{\lambda_{2}}{\mu_{2,0}}+\frac{\lambda_{2,1}}{\mu_{2,1}}+\frac{\lambda_{2,2}}{\mu_{2,2}}<1,
$$

where $\mu_{2}$ denotes the average service rate of $S_{2}$.

\section{B. Stability condition for relay}

The arrival process of the packet in $S_{1}$ that transmits successfully only to the relay is:

$$
A_{1} \cap\left\{Q_{1,0} \neq 0\right\} \cap O_{S_{1} R} \cap \overline{O_{S_{1} D_{2}}} \cap \overline{O_{S_{1} D_{2}}} .
$$

Since the arrival process to the relay is stationary, the expected arrival rate of $R_{0}$ from $S_{1}$ and $S_{2}$ are respectively

$$
\begin{aligned}
& \lambda_{R, 0,1}=\frac{\lambda_{1}}{1-P_{1,3}} P_{S_{1} R}\left(1-P_{S_{1} D_{1}}\right)\left(1-P_{S_{1} D_{2}}\right), \\
& \lambda_{R, 0,2}=\frac{\lambda_{2}}{1-P_{2,3}} P_{S_{2} R}\left(1-P_{S_{2} D_{1}}\right)\left(1-P_{S_{2} D_{2}}\right),
\end{aligned}
$$

where $P_{2,3}=\left(1-P_{S_{2} D_{1}}\right)\left(1-P_{S_{2} D_{2}}\right)\left(1-P_{S_{2} R}\right)$. Therefore $R_{0}$ has arrival rate $\lambda_{R_{0}}=\lambda_{R, 0,1}+\lambda_{R, 0,2}$.

The service process of $R_{0}$ consists of three parts: i.) $R_{0}$ transmits successfully to $D_{1}$ and $D_{2}$; ii.) Packet in $R_{0}$ transmits only successfully to $D_{1}$ and is moved to $R_{1}$; ii.) Packet in $R_{0}$ transmit only successfully to $D_{2}$ and is moved to $R_{2}$. So the event of service process of $R_{0}$ is

$$
\begin{aligned}
A_{1} \cap\left\{Q_{1}=0\right\} \cap & \left(O_{R D_{1}} \cup O_{R D_{2}}\right) \\
& +A_{2} \cap\left\{Q_{2}=0\right\} \cap\left(O_{R D_{1}} \cup O_{R D_{2}}\right) .
\end{aligned}
$$


Hence the service rate of $R_{0}$ is

$$
\begin{aligned}
\mu_{R_{0}}=\left[\omega_{1}\left(1-\frac{\lambda_{1}}{\mu_{1}}\right)+\omega_{2}\left(1-\frac{\lambda_{2}}{\mu_{2}}\right)\right] \\
\times\left(P_{R D_{1}}+P_{R D_{2}}-P_{R D_{1}} P_{R D_{2}}\right) .
\end{aligned}
$$

So the stability condition of $R_{0}$ is $\lambda_{R_{0}}<\mu_{R_{0}}$.

There are three cases for $R_{1}$ : i.) Packet in $S_{1}$ transmits successfully to both relay and $D_{1}$; ii.) $F_{1}$ packet transmits successfully to the relay; iii.) Packet in $R_{0}$ transmits successfully to $D_{1}$ and is moved to $R_{1}$.

i) The arrival process of the packet in $S_{1}$ that transmits successfully to the relay and $D_{1}$ is: $A_{1} \cap\left\{Q_{1,0} \neq 0\right\} \cap$ $O_{S_{1} D_{1}} \cap O_{S_{1} R} \cap \overline{O_{S_{1} D_{2}}}$. Then the arrival rates

$$
\begin{aligned}
\lambda_{R, 1,1}= & \frac{\lambda_{1}}{1-P_{1,3}} P_{S_{1} R} P_{S_{1} D_{1}}\left(1-P_{S_{1} D_{2}}\right) \\
& +\frac{\lambda_{2}}{1-P_{2,3}} P_{S_{2} R} P_{S_{2} D_{1}}\left(1-P_{S_{2} D_{2}}\right),
\end{aligned}
$$

where $P_{2,3}=\left(1-P_{S_{2} D_{1}}\right)\left(1-P_{S_{2} D_{2}}\right)\left(1-P_{S_{2} R}\right)$.

ii) The arrival process of the $F_{1}$-packet transmits successfully to $R$ and becomes $F_{R_{1}}$-packets is $X_{1,1}=$ $A_{1} \cap\left\{Q_{1,1} \neq 0\right\} \cap O_{S_{1} R} \cap \overline{O_{S_{1} D_{2}}}$, where $\left\{Q_{1,1} \neq 0\right\}$ denotes the event of the source node transmitting the $F_{1}$-packet. By using Little's theorem, the probability of $\left\{Q_{1,1} \neq 0\right\}$ is $\frac{\lambda_{1,1}}{\mu_{1,1}}$. So the arrival rate of the $F_{2}$-packet of $S_{1}$ into $R_{1}$ is

$$
\frac{\lambda_{1}}{1-P_{1,3}} \cdot \frac{P_{S_{1} D_{1}}\left(1-P_{S_{1} R}\right)\left(1-P_{S_{1} D_{2}}\right)}{P_{S_{1} R}+P_{S_{1} D_{2}}-P_{S_{1} R} P_{S_{1} D_{2}}} .
$$

Hence the arrival rate of the $F_{1}$-packet from $S_{1}$ and $S_{2}$ into $R_{1}$ can be calculated as

$$
\begin{aligned}
\lambda_{R, 1,2}=\frac{\lambda_{1}}{1-P_{1,3}} & P_{S_{1} D_{1}}\left(1-P_{S_{1} R}\right)\left(1-P_{S_{1} D_{2}}\right) \\
& \times \frac{P_{S_{1} R}\left(1-P_{S_{1} D_{2}}\right)}{P_{S_{1} R}+P_{S_{1} D_{2}}-P_{S_{1} R} P_{S_{1} D_{2}}} \\
+\frac{\lambda_{2}}{1-P_{2,3}} & P_{S_{2} D_{1}}\left(1-P_{S_{2} R}\right)\left(1-P_{S_{2} D_{2}}\right) \\
& \times \frac{P_{S_{2} R}\left(1-P_{S_{2} D_{2}}\right)}{P_{S_{2} R}+P_{S_{2} D_{2}}-P_{S_{2} R} P_{S_{2} D_{2}}} .
\end{aligned}
$$

iii) The arrival process for the packet in $R_{0}$ that transmits successfully to $D_{1}$ and is moved to $R_{1}$, is $A_{1} \cap\left\{Q_{1}=\right.$ $0\} \cap\left\{Q_{R_{0}} \neq 0\right\} \cap O_{R D_{1}} \cap \overline{O_{R D_{2}}}$, where $\left\{Q_{R_{0}} \neq 0\right\}$ denotes the event that the $R_{0}$ is not empty, which probability, by using Little's theorem, is $\frac{\lambda_{R_{0}}}{\mu_{R_{0}}}$. So the arrival rate is

$$
\begin{aligned}
\lambda_{R, 1,3} & =\omega_{1}\left(1-\frac{\lambda_{1}}{\mu_{1}}\right) \frac{\lambda_{R_{0}}}{\mu_{R_{0}}} P_{R D_{1}}\left(1-P_{R D_{2}}\right) \\
& +\omega_{2}\left(1-\frac{\lambda_{2}}{\mu_{2}}\right) \frac{\lambda_{R_{0}}}{\mu_{R_{0}}} P_{R D_{1}}\left(1-P_{R D_{2}}\right),
\end{aligned}
$$

By (13), we have

$\lambda_{R, 1,3}=\lambda_{R_{0}}\left(P_{R D_{1}}+P_{R D_{2}}-P_{R D_{1}} P_{R D_{2}}\right) P_{R D_{2}}\left(1-P_{R D_{1}}\right)$
Combining the three cases, $R_{1}$ has arrival rate

$$
\lambda_{R_{1}}=\lambda_{R, 1,1}+\lambda_{R, 1,2}+\lambda_{R, 1,3} .
$$

By Strategy 1, only when the time slot is assigned to $S_{1}$ $\left(S_{2}\right)$, and $S_{1}\left(S_{2}\right)$ and $R_{0}$ are empty, $R_{1}$ has chance to transmit. If $R_{2}$ has packet, $R_{1}$ and $R_{2}$ can do network coding; while if $R_{2}$ is empty, $R_{1}$ will transmit by itself. For the aforementioned two cases, $R_{1}$ will have the same service process and the same service rate. Specifically, the service process of $R_{1}$ is

$$
\begin{aligned}
A_{1} \cap\left\{Q_{1}\right. & =0\} \cap\left\{Q_{R_{0}}=0\right\} \cap O_{R D_{2}} \\
& +A_{2} \cap\left\{Q_{2}=0\right\} \cap\left\{Q_{R_{0}}=0\right\} \cap O_{R D_{2} .}
\end{aligned}
$$

So the service rate of $R_{1}$ is

$$
\mu_{R_{1}}=\left[\omega_{1}\left(1-\frac{\lambda_{1}}{\mu_{1}}\right)+\omega_{2}\left(1-\frac{\lambda_{2}}{\mu_{2}}\right)\right]\left(1-\frac{\lambda_{R_{0}}}{\mu_{R_{0}}}\right) P_{R D_{2}} .
$$

Hence the stability condition of $R_{1}$ is $\lambda_{R_{1}}<\mu_{R_{1}}$.

Similar as $R_{1}, R_{2}$ has arrival rate

$$
\lambda_{R, 2}=\lambda_{R, 2,1}+\lambda_{R, 2,2}+\lambda_{R, 2,3},
$$

The service rate of $R_{2}$ is

$$
\mu_{R_{2}}=\left[\omega_{1}\left(1-\frac{\lambda_{1}}{\mu_{1}}\right)+\omega_{2}\left(1-\frac{\lambda_{2}}{\mu_{2}}\right)\right]\left(1-\frac{\lambda_{R_{0}}}{\mu_{R_{0}}}\right) P_{R D_{1}} .
$$

Therefore the stability condition of $R_{2}$ is $\lambda_{R_{2}}<\mu_{R_{2}}$. In summary, we conclude that the stability conditions for strategy 1 are

$$
\left\{\begin{array}{l}
\frac{\lambda_{1}}{\mu_{1,1}}+\frac{\lambda_{1,2}}{\mu_{1,2}}+\frac{\lambda_{1,3}}{\mu_{1,3}}<1, \\
\frac{\lambda_{2}}{\mu_{2,1}}+\frac{\lambda_{2,2}}{\mu_{2,2}}+\frac{\lambda_{2,3}}{\mu_{2,3}}<1, \\
\lambda_{R_{0}}<\mu_{R_{0}}, \\
\lambda_{R_{1}}<\mu_{R_{1}}, \\
\lambda_{R_{2}}<\mu_{R_{2}} .
\end{array}\right.
$$

\section{STABility Analysis For Strategy 2 AND 3}

In this strategy, the Similarly, the stability condition for strategy 2 is

$$
\left\{\begin{array}{l}
\frac{\lambda_{1}}{\mu_{1,1}}+\frac{\lambda_{1,2}}{\mu_{1,2}}+\frac{\lambda_{1,3}}{\mu_{1,3}}<1, \\
\frac{\lambda_{2}}{\mu_{2,1}}+\frac{\lambda_{2,2}}{\mu_{2,2}}+\frac{\lambda_{2,3}}{\mu_{2,3}}<1, \\
\lambda_{R_{0}}^{\prime}<\mu_{R_{0}}^{\prime} \\
\lambda_{R_{1}}^{\prime}<\mu_{R_{1}}^{\prime}, \\
\lambda_{R_{2}}^{\prime}<\mu_{R_{2}}^{\prime} .
\end{array}\right.
$$

The stability condition of strategy 3 is

$$
\frac{\lambda_{R_{0}}^{\prime \prime}}{\mu_{R_{0}}^{\prime \prime}}+\frac{\lambda_{R_{1}}^{\prime \prime}}{\mu_{R_{1}}^{\prime \prime}}+\frac{\lambda_{R_{2}}^{\prime \prime}}{\mu_{R_{2}}^{\prime \prime}}<1 .
$$

Moreover, we have the following interesting result for the three strategies.

Theorem 1: The stability region of strategy 1 is bigger than that of strategy 2 , and the stability region of strategy 2 is bigger )than that of strategy 3 .

Proof: Omitted due to limited space. 


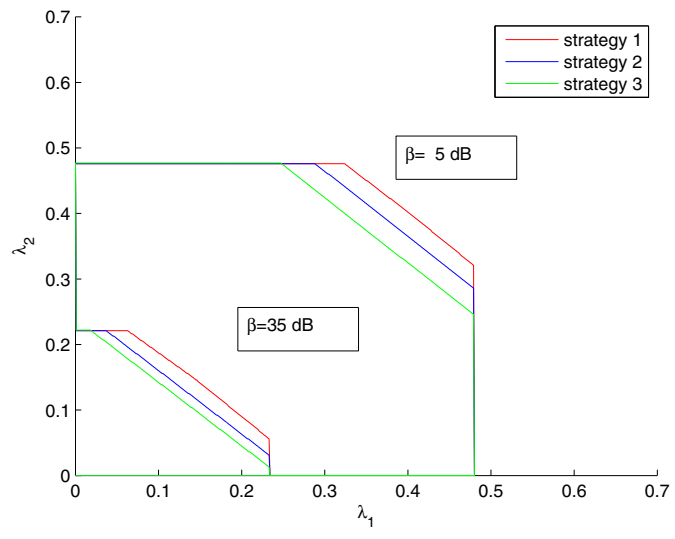

Fig. 2. Stability region under $\beta=5$ and $\beta=35 \mathrm{~dB}$. $\left(\omega_{1}=\omega 2=1 / 2\right)$

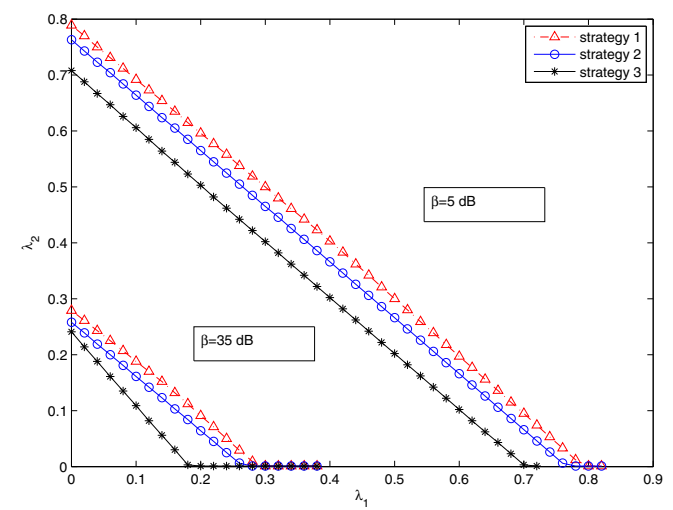

Fig. 3. Maximum stable throughput under $\beta=5 \mathrm{~dB}$ and $\beta=35 \mathrm{~dB}$.

\section{Numerical Results}

In our Monte-Carlo simulations, parameters are symmetrically selected as $\gamma_{S_{1} R}=\gamma_{S_{2} R}=40 \mathrm{~dB}, \gamma_{S_{1} D_{1}}=\gamma_{S_{2} D_{2}}=$ $20 \mathrm{~dB}, \gamma_{S_{1} D_{2}}=\gamma_{S_{2} D_{1}}=25 \mathrm{~dB}$, and $\gamma_{R D_{1}}=\gamma_{R D_{2}}=50 \mathrm{~dB}$.

Fig. 2 shows the stability regions of the three strategies for $\omega_{1}=\omega_{2}=1 / 2$. We can observe that the strategy 1 and 2 with network coding have larger stability region, and the stability region for strategy 1 is the largest, which validate the theoretical prediction. We can also observe that the stability region for the three strategies become smaller as the required SNR threshold $\beta$ increases.

Fig. 3 shows the maximum stability regions of the three strategies. We can observe that the maximum stability regions are larger than the corresponding stability regions for $\omega_{1}=$ $\omega_{2}$. Specifically, Regions in Fig. 3 enlarges the corresponding regions in Fig 2 in the areas where either of $\lambda_{1}$ or $\lambda_{2}$ is small.

\section{CONCLUSION}

In this paper, we propose an opportunistic relay with regenerative network coding for the multicast cell. Specifically, we propose three relaying transmission strategies with and without network coding. By analyzing the stability region for the three strategies, we demonstrate that the strategies with opportunistic network coding enjoy large stability regions. In addition, the strategy with opportunistic network coding outperforms that with relative static network coding. By simulation, we also find that the stability regions for the three strategies become smaller as the required received SNR threshold increases.

\section{ACKNOWLEDGEMENT}

This work is supported by NSF China \#60972031, by SEU SKL project \#W200907, by Huawei Funding \#YJCB2009024WL and \#YJCB2008048WL, and by National 973 project \#2009CB824900.

\section{REFERENCES}

[1] I. F. Akyildiz, Y. Altunbasak, F. Fekri, and R. Sivakumar, "AdaptNet: adaptive protocol suite for next generation wireless internet" in IEEE Communications Magazine, vol. 42, pp. 128-138, Jan. 2004.

[2] J. Mitola. III, "Cognitive Radio for Flexible Mobile Multimedia Communications ," IEEE 1999 Mobile Multimedia Conference (MoMuC), pp. 3-10, Nov. 1999.

[3] S. Haykin "Cognitive radio: brain-empowerd wireless communications," IEEE Jounal on Selected Areas in Communications, vol. 23, no. 2, pp. 201-220, Jan. 2005.

[4] N. Devroye, P. Mitran, and V. Tarokh, "Achievable rates in cognitive radio," IEEE Trans. Inf. Theory, vol. 52, no. 5, pp. 1813-1827, May. 2006.

[5] A. K. Sadek, K. J. Ray Liu, and A. Ephremides, "Cognitive Multiple Access Via Cooperation: Protocol Design and Performance Analysis," IEEE Trans. Inf. Theory, vol. 53, no. 10, pp. 3677-3696, Oct., 2007.

[6] R. Ahlswede, N. Cai, S.-Y. R. Li, and R. W. Yeung, "Network infomation flow," IEEE Trans. Inf. Theory, vol. 46, no. 4, pp. 1204-1216, Jul., 2000.

[7] F. Xue, L.-L. Xie, and P. R. Kumar, "The Transport Capacity of Wireless Networks Over Fading Channels," IEEE Trans. Inf. Theory, pp. 834-847, Mar., 2005.

[8] Y. E. Sagduyu, and A. Ephremides "Cross-Layer Optimization of MAC and Network Coding in Wireless Queueing Tandem Networks," IEEE Trans. Inf. Theory, vol. 54, no. 2, pp. 554-571, Feb., 2008.

[9] J. Li, and W. Chen "Power Allocation in the High SNR Regime for a Multicast Cell with Regenerative Network Coding," IEEE Communications Letters, vol. 13, no. 4, pp. 271-273, 2009.

[10] R. Urgaonkar, and M. J. Neely, "Opportunistic Scheduling with Reliability Guarantees in Cognitive Radio Networks," Proc. IEEE Infocom, pp. 1301-1309, Apr., 2008.

[11] S. Huang, X. Liu, and Z. Ding, "Opportunistic Spectrum Access in Cognitive Radio Networks," Proc. IEEE Infocom, pp. 1427-1435, Apr., 2008.

[12] K.Yang, and X. Wang "Cross-Layer Network Planning for Multi-Radio Multi-Channel Cognitive Wireless Networks,'IEEE Trans. Communication, vol. 56, no. 10, pp. 1705-1714, Oct., 2008.

[13] G. Ganesan, and Y. Li, "Cooperative Spectrum Sensing in Cognitive Radio, Part I: Two User Networks,' IEEE Trans. Wireless Communication, vol. 6, no. 6, pp. 2204-2213, Jul., 2007.

[14] O. Simeone, Y. Bar-Ness, and U. Spagnolini "Stable Throughput of Cognitive Radios With and Without Relaying Capability," IEEE Trans. Communication, vol. 55, no. 12, pp. 2351-2360, Dec., 2007.

[15] R. Rao, and A. Ephremides "On the stability of interacting queues in a multi-access systems," IEEE Trans. Inf. Theory, vol. 34, no. 5, pp. 918930, Sep., 1988. 